Die Hirntod-

diagnostik

gewährleistet die

Sicherheit der

korrekten

Todesfeststellung. sprache durch Pflegekräfte, was nicht dem Umgang mit „echten" Leichen entspricht. Damit existieren Hirntote für die Angehörigen und Pflegekräfte zumindest im sozialen Kontext vorübergehend weiter.

\section{Kritik an der Qualität der Hirntoddiagnostik}

Immer wieder wird die Qualität der Hirntoddiagnostik öffentlichkeitswirksam in Frage gestellt. Jedoch ist kein Fall bekannt, bei dem ein Patient unrichtigerweise für hirntot erklärt oder eine Organentnahme erfolgte, nachdem eine komplette, formal korrekte Hirntoddiagnostik durchgeführt wurde. Im Rahmen der Spenderevaluation im Entnahmekrankenhaus vor Ort wird die vorliegende Hirntoddiagnostik von Mitarbeitern der Deutschen Stiftung für Organspende, aber auch vom Entnahmechirurgen unmittelbar vor einer Spender-OP noch einmal auf formale Gesichtspunkte hin überprüft. Kommt es bei dieser Überprüfung zu Zweifeln am korrekten Vorgehen oder fallen formale Fehler auf (z.B. das Fehlen einer Unterschrift), wird die Organspende, auch unter Inkaufnahme des Spenderorganverlustes, nicht durchgeführt.

Die Hirntoddiagnostik gewährleistet die Sicherheit der korrekten Todesfeststellung. Sie identifiziert aber auch Patienten, bei denen eben nicht ein kompletter Verlust der Hirnaktivität vorliegt. Diese Fälle stellen jedoch keinen Qualitätsmangel dar, sondern sind ein Indiz für ein funktionierendes System mit mehreren voneinander unabhängigen Sicherheitsbarrieren.

\section{Fazit für die Pflege}

- Das Feststellen des Hirntods beinhaltet den sicheren Nachweis eines irreversiblen Verlustes aller Hirnfunktionen.

- Werden die organerhaltenden Maßnahmen beendet, folgt dem Hirntod innerhalb von wenigen Minuten der Herz-Kreislauf-Stillstand und der klinische Tod.

- Das Hirntodkonzept wurde primär entwickelt, um den Todeszeitpunkt von Patienten mit schwersten Hirnschädigungen unter Bedingungen der modernen Intensivmedizin festzustellen. Die Feststellung des Hirntodes ist heute auch die Voraussetzung, um Organspender sein zu können ("dead donor rule").

\section{Literatur beim Verfasser}

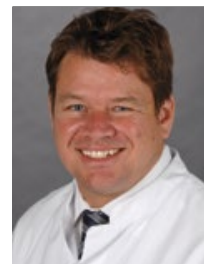

Prof. Dr. med. Markus Guba

Klinik für Allgemeine, Viszeral-, Transplantations-, Gefäß- und Thoraxchirurgie

Klinikum der Universität München Klinikum Großhadern

Marchioninistr. 15

81377 München

Markus.Guba@med.uni-muenchen.de

\title{
Transplantationsregister-Gesetz: AWMF fordert Nachbesserungen
}

\section{Der vom Bundesgesundheitsministeri- um vorgelegte Entwurf eines Trans- plantationsregister-Gesetzes, das die einheitliche und umfassende Erhe- bung aller transplantationsmedizini- schen Daten regelt, stößt bei der Arbeitsgemeinschaft der Wissen- schaftlichen Medizinischen Fachge- sellschaften (AWFM) auf breite Zustimmung. Allerdings fordert die AWFM auch wichtige Ergänzungen.}

Nachbesserungen des Gesetzesentwurfs fordert die AWFM vor allem für die Regelungen zum Beirat. Diesem müssten gesetzlich verankert auch Vertreter der wissenschaftlichen medizinischen Fachgesellschaften angehören, um deren Sachverstand stärker als bisher in das Vorhaben einfließen zu lassen. Neben der im Gesetzentwurf explizit aufgeführten Deutschen Transplantati- onsgesellschaft sollten auch weitere Fachgesellschaften einbezogen werden, die mit der Transplantationsindikation, -durchführung und Nachbetreuung befasst sind.

Dazu zähle beispielsweise die Deutsche Gesellschaft für Gastroenterologie, Verdauungs- und Stoffwechselkrankheiten (DGVS): „Das Register wird die Entwicklung der Transplantationsmedizin in Deutschland sicherlich begünstigen", sagt DGVS-Experte Prof. Dr. med. Thomas Berg vom Universitätsklinikum Leipzig. Allerdings weise der Gesetzesentwurf aus klinisch-wissenschaftlicher Sicht Lücken auf, etwa bei der Spenderbehandlung: Bislang ist nicht geklärt, wie ein Verstorbener zu behandeln ist, damit seine Organe in bestmöglichem Zustand für die Transplantation verfügbar bleiben. Zudem spricht sich die
DGVS dafür aus, die Datenerhebung zur Nachsorge auf Transplantationszentren zu beschränken.

Die Zahl der Organspender in Deutschland stagniert auf niedrigem Niveau: Seit 2013 liegt sie konstant unter 900 . Im Jahr 2010 fanden sich noch 1.300 Menschen, deren Organe nach ihrem Tod transplantiert werden durften. Ein Transplantationsregister soll die Situation verbessern und Manipulationen vorbeugen. Eine Registerstelle soll künftig alle transplantationsmedizinischen Daten zentral erheben, speichern und prüfen.

Das Gesetz soll noch in diesem Jahr verabschiedet werden.

www.awmf.org 